Estudios Fronterizos, vol. 10, núm. 19, enero-junio 2009, pp. 183-208

\title{
Integración económica en América del Norte: lección de la experiencia de la Unión Europea para el TLCAN ${ }^{1}$
}

\author{
Ramón de Jesús Ramírez Acosta* \\ Ramón A. Castillo Ponce ${ }^{* *}$
}

Resumen. En el presente documento se analiza, con base en estudios de diversos autores, el grado de sincronización económica de los países de Europa Central y Oriental con respecto a la Unión Europea en el periodo inmediato anterior a las adhesiones del año 2004. Posteriormente, se determina de manera formal el grado de sincronización económica en América del Norte para el periodo de transición del TLCAN. En particular, se estiman modelos de cointegración y características comunes de corto plazo para los PIBs de Canadá, México y Estados Unidos. Encontramos que las economías de la región han alcanzado un grado significativo de integración. Con base en estos resultados, y a los hechos identificados para el caso europeo, se esbozan las posibilidades de una evolución del TLCAN hacia una integración económica más profunda.

Palabras clave: sincronización económica, integración económica avanzada, cointegración, ciclo común.

Abstract. In this document we analyze, based on previous work from various authors, the degree of economic synchronization between Central and Eastern European countries and the European Union prior to the 2004 adhesion treaties. Then, we formally determine the degree of economic synchronization in North America for the NAFTA transitional period. In particular, we perform cointegration and common features tests for the GDPs of Canada, Mexico and the US. We find that the economies of the region are highly integrated. Based on these results, and the facts identified for the European case, we discuss some possibilities for the evolution of NAFTA into a deeper form of economic integration.

Keywords: economic synchronization, advanced economic integration, cointegration, common cycles.

\footnotetext{
${ }^{1}$ Agradecemos los comentarios y las sugerencias de Pablo Mejía y los participantes en el XVIII Coloquio Mexicano de Economía Matemática y Econometría. La acotación usual aplica.

*Profesor de la Facultad de Economía y Relaciones Internacionales de la Universidad Autónoma de Baja California. Correo electrónico: rramirez@uabc.mx **Universidad Autónoma de Baja California y California State University, Los Ángeles. Correo electrónico: racastill@uabc.mx
} 


\section{Introducción}

El pasado 31 de diciembre del año 2007 llegó a su fin el periodo de transición del Tratado de Libre Comercio de América del Norte (TLCAN), es decir, se alcanzó la categoría de zona de libre comercio; salvo en algunos sectores específicos como el de automóviles. En este contexto, en términos de las relaciones comerciales en América del Norte, el tema sobre el cual se centrará el debate será sin duda la conformación de la siguiente etapa en el proceso de integración económica regional.

Para México, la cuestión requiere reflexionarse en función de sus relaciones económicas con Estados Unidos y Canadá; dentro de un futuro de corto plazo, sobre todo en la respuesta a dos preguntas: ¿existe un grado de sincronización importante del ciclo de negocios regional en América del Norte para establecer una etapa más avanzada de integración económica regional?, y ¿qué lección de la experiencia de la Unión Europea (UE) puede obtenerse para el caso del TLCAN?

El documento que a continuación se presenta consta de tres secciones. En la primera se realiza una revisión de literatura sobre el caso europeo, para determinar el grado de la sincronización económica de los Países de Europa Central y Oriental (PECOS) con respecto a la UE; en el marco de referencia de lo que se ha denominado la "quinta ampliación" del año 2004. El propósito es puntualizar la asociación que existe entre la homologación de los ciclos económicos y la integración económica de países o regiones. En la segunda, se determina el grado de sincronización económica en América del Norte utilizando un análisis econométrico, con el objetivo de evaluar la posibilidad de avanzar en el proceso de integración económica hacia una forma más profunda de intercambio comercial. Cabe mencionar que nuestra intención no es equiparar la experiencia de la UE en su proceso de integración económica con América del Norte, sino extraer de dicho proceso alguna lección para los países miembros del TLCAN; esto se lleva a cabo en la tercera sección. En ésta misma se establecen las líneas futuras de investigación sobre el tema y la conclusión. 
RAMÓN DE JESÚS RAMíREZ ACOSTA/INTEGRACIÓN ECONÓMICA EN AMÉRICA DEL NORTE

\section{La quinta ampliación de la Unión Europea}

\section{Acuerdos de asociación}

En términos de integración económica regional, la UE es indudablemente hoy en día la experiencia a investigar y el marco de referencia de análisis para todo intento contemporáneo en este sentido. Dentro de esta experiencia destaca por su importancia su quinta ampliación, caracterizada por la firma de diez tratados de adhesión en el mes de mayo del año 2004. ${ }^{2}$ Estos países se conocen en la literatura como los PECOS. En este contexto, la comunidad europea pasó a ser conocida de UE-15 a UE-25, en referencia al número de países integrantes. En la actualidad cuenta con 27 miembros formales al sumarse Rumania y Bulgaria el 1 de enero de 2007. A esta etapa se le considera la sexta ampliación y ahora se le conoce como la UE-27.

El primer objetivo de nuestro análisis es intentar establecer si previo a la quinta ampliación existió la sincronización de los ciclos económicos de estos países con relación a la entonces UE-15, es decir, durante el periodo que comprende la década de los noventa. Es importante mencionar que esta adhesión tiene un antecedente importante que de alguna manera recuerda al TLCAN: los acuerdos de asociación.

Los acuerdos de asociación se firmaron a principios de la década de los noventa entre los diez países candidatos y la UE-15, con el propósito implícito de establecer la integración una vez cumplidos los criterios planteados en éstos. Para tal efecto, la UE-15 puso en marcha el programa comunitario PHARE, creado para dotar de apoyo financiero los esfuerzos por reformar y reconstruir las economías y para fomentar la transición política. Cabe señalar que en este sentido, los acuerdos contrastan con lo estipulado en el TLCAN, ya que en este último no se considera la posibilidad de ayuda financiera.

Durante los años noventa, la UE-15 y los países adherentes fueron concluyendo progresivamente los acuerdos de asociación, también

\footnotetext{
${ }^{2}$ Los países participantes fueron: Chipre, la República Checa, Estonia, Hungría, Letonia, Lituania, Malta, Polonia, la República Eslovaca y Eslovenia
} 
conocidos como los "Acuerdos Europeos". Estos acuerdos proporcionaron una base legal y económica al proceso, ya que se tuvieron que cumplir sobre todo con condiciones económicas en el contexto de lo que se denominó Criterios de Copenhague. ${ }^{3}$

La sincronización económica, preadhesión de los PECOS

El análisis de la sincronización de los ciclos económicos de los países adherentes a la UE-15, fue estudiado fundamentalmente a partir del año 2000 en el contexto de la evaluación de sus candidaturas. Por ejemplo, Darvas y Szapáry (2004) derivan que durante la etapa de los acuerdos de asociación solamente en los países más grandes en términos de su producto interno bruto (PIB) como son Polonia y Hungría, se observó cierta sincronización en sus ciclos económicos con la UE-15, en tanto que en los demás se registró poca o nula sincronización. De la misma manera, encuentran que la mayor sincronización se dio con la zona del Euro. Tomando como base a 8 países de los PECOS, 10 de la zona del Euro y 3 fuera de ésta, analizan una serie de tiempo del PIB por diez años, de 1993 a 2002, y establecen la correlación por medio del análisis de comovimientos.

Por su parte, Guisan, Aguayo y Carballas (2004) desagregan una serie de tiempo de larga duración desde el año 1950 hasta el 2002, para establecer la relación del crecimiento económico y los ciclos entre países de la UE-15 con los PECOS, por medio del comportamiento del índice de producción industrial. El análisis lo realizan dividiendo este periodo largo en 5 etapas específicas que son de 1950 a 1960, de 1960 a 1975, de 1975 a 1985, de 1985 a 1991 y de 1991 a 2002. Los autores se enfocan en los casos especiales de Polonia y Hungría con respecto a países seleccionados de la UE-15 especialmente con España. Los resultados muestran que la relación cambia con intensidad variable en el tiempo y en el espacio, pero es significativamente más estrecha en el periodo previo a la adhesión, es decir, en la etapa de

\footnotetext{
${ }^{3}$ Fueron tres. El primero establecía la generación de instituciones estables que garantizaran la democracia y el Estado de derecho. El segundo el desarrollo de una economía de mercado. El tercero la adopción de todo el acervo comunitario de la Unión Europea.
} 
1991 a 2002, lo cual se deduce que fue originado por la firma de los acuerdos de asociación de 1991.

En la misma dirección de análisis, Firdmuc y Korhonen (2004) estiman que actualmente los PECOS se encuentran ya en lo que puede considerarse un alto grado de sincronización económica con la zona europea del Euro. Más aun, vuelve a encontrarse como en otros estudios que Polonia y Hungría son los países de los PECOS más avanzados en este aspecto, de manera tal que incluso se asegura que cumplen ya con la condición fundamental de sincronización. Para llegar a lo anterior, los autores revisaron 27 estudios independientes realizados del año 2000 al 2004, de donde extrajeron 400 estimaciones de correlación de ciclos económicos entre la zona del Euro y los PECOS, basados en diversos métodos econométricos.

Por su parte, Eickmeier y Breitung (2005), de igual manera, investigan la sincronización del ciclo económico de los PECOS pero solamente con la zona del Euro. Para tal efecto, centran su atención en el análisis de transmisión de los "shocks" económicos para de esta manera determinar la sincronización. Como era de esperarse, encuentran que la transmisión de la zona del Euro a los PECOS tendió a incrementarse en el tiempo, sobre todo en el periodo que comprende de 1993 a 2003. Se utiliza en esta investigación un modelo de factores dinámicos de largo plazo sujeto a restricciones estructurales comunes.

Recientemente WoŸniak y Paczyñski (2007), en un estudio realizado en el contexto de las propuestas para determinar la posible incorporación de nuevos miembros a la zona del Euro, establecen que el grado de sincronización económica de los países de la quinta ampliación con esta zona es lo suficientemente fuerte para recomendar su adhesión. De hecho, de los 15 miembros actuales tres de éstos, Chipre, Malta y Eslovenia, pertenecen a los PECOS. Los autores utilizan series de tiempo del PIB para establecer los comovimientos con base en un filtro de Kalman.

Acuerdos de asociación y TLCAN

La sincronización del ciclo de negocios de los PECOS y de la UE-15, sobre todo de los países más importantes en el orden económico, se 
apoyó fundamentalmente en la implementación de relaciones económicas y comerciales. Los capítulos que integraron la parte económica de los acuerdos tenían como objetivo, al igual que el TLCAN, la creación de una zona de libre comercio caracterizada por el desarme arancelario de bienes y servicios.

Dentro de estos capítulos, además de las mercancías, destacó el de los servicios y en especial la presencia de personas físicas y el transporte transfronterizo. Otros capítulos importantes fueron las inversiones que regularon los movimientos de capital, las salvaguardas, la propiedad intelectual, la contratación pública que reglamentaba la participación de los gobiernos en el comercio, las normas de origen aplicables a los productos originarios, la solución de diferencias para tratar de evitar la adopción de medidas unilaterales de defensa y acuerdos específicos de carácter sectorial y preferencial con periodo de transición diferente. Como se puede observar, el objetivo y contenido de los acuerdos de asociación europea, en el escenario económico, fue prácticamente similar al TLCAN. ${ }^{4}$

Al terminar el periodo de transición de los acuerdos de asociación (1991-2004), la Comisión Europea consideró y declaró que los notables progresos conseguidos por los países adherentes en sus reformas económicas, así como en sus estructuras administrativas y judiciales, los hacían sujetos de adhesión como miembros formales, con lo cual iniciaron una fase más profunda de integración económica. Con estas ideas en mente, en el mes de mayo de 2004 se firmaron los Tratados de Adhesión generando la mayor ampliación que haya experimentado la UE en su historia, tanto en sus dimensiones geográficas y económicas como en la diversidad de culturas con diez nuevos países.

Ahora bien, a partir del criterio de que la integración económica es un proceso, el cual pasa por diversas etapas en forma paulatina y progresiva, es posible establecer una clasificación donde cada una de las etapas tiene ciertos rasgos esenciales que la distinguen tanto de la eta-

${ }^{4}$ Para una comparación exhaustiva puede consultarse para los acuerdos de asociación la página web http://www.europarl.europa.eu/factsheets/6_3_3_es.htm del Parlamento Europeo, y para el TLCAN el Resumen del Tratado de Libre Comercio de América del Norte, publicado por el Sistema de Información del Comercio Exterior en http://www.sice.oas.org/trade/nafta/naftatce.asp 
pa inmediata anterior como de la posterior. En este sentido, existe un consenso más o menos amplio en la literatura que trata el tema respecto a cuáles son las etapas por las que pasan los procesos de integración. Para efectos de este documento, al margen de complejidades teóricas, se considera la clasificación tradicional en área de preferencias arancelarias, zona de libre comercio, unión aduanera, mercados comunes y unión económica. Podemos así señalar que la integración económica profunda se inicia a partir de los esfuerzos de creación de uniones aduaneras.

La unión aduanera implica a su vez un subproceso de la integración económica en el que dos o más territorios aduaneros forman uno solo, caracterizado por una liberalización de los flujos comerciales por medio de la desgravación arancelaria de la fase anterior; además de adoptar frente a terceros países una política de tarifa externa común y la distribución de los ingresos aduaneros entre sus miembros conforme a una fórmula acordada. Esto último implica de hecho la aparición de una política comercial común.

Es conveniente mencionar que en la actualidad, una de las medidas más importantes que los PECOS han tenido que adecuar para acelerar su integración económica es la relacionada con la integración aduanera. En los acuerdos de asociación europeos se tuvo especial interés en establecer medidas para la adaptación del sistema aduanero de los países adherentes al sistema que funcionaba en la UE. De esta manera, todos los sistemas de aduanas se han visto en la necesidad de adaptarse a los vigentes en la UE y a la introducción de procedimientos uniformes a los estándares europeos, dando pasos muy importantes para adecuarse a la unión aduanera europea dentro de un proceso de integración económica profunda. ${ }^{5}$

Este subproceso no ha sido fácil en la realidad y los retos que se imponen son extraordinarios, ya que los Estados que integran una unión aduanera, además de reducir las tarifas aduaneras entre sí hasta llegar a cero propias de una zona de libre comercio, deben de adoptar un arancel externo común en forma gradual con plazos y listas

\footnotetext{
${ }^{5}$ Para una información más amplia sobre este proceso puede consultarse la página web http://europa.eu/pol/cust/index_es.htm
} 
temporarias de excepciones, que normalmente son diferentes según el desarrollo económico de los países integrantes en relación a los productos que importan de países de fuera de la región.

$\mathrm{Al}$ existir un arancel externo común, se eliminan las reglas de origen, por lo que un bien de procedencia extranjera ingresado legalmente por cualquier aduana de la unión, previo pago de la tarifa común que se haya fijado, tiene libre circulación por el espacio geográfico de los países socios. Otro dato importante de mencionar es respecto de la forma de negociación con el exterior, la cual debe hacerse necesariamente en bloque, con lo cual se entra de lleno en la primera acción de política económica común en el escenario comercial.

El crecimiento económico de los PECOS durante el periodo de los acuerdos de asociación

Durante la década de los noventa se firmaron de manera progresiva los acuerdos de asociación con los PECOS. Los primeros se firmaron en el año de 1991 con Hungría y Polonia. En 1993 se realizaron con Eslovaquia y la República Checa, mientras que en 1995 fue con Estonia, Letonia y Lituania, y por último en 1996 con Eslovenia.

En términos de su crecimiento económico, puede afirmarse que el funcionamiento de dichos acuerdos significó para algunos de los países involucrados un periodo de gran impulso y para otros de estabilidad. Por ejemplo, de acuerdo con los datos del cuadro 1, para Hungría, Letonia y Lituania implicó superar altas tasas negativas hacia tasas positivas. Destaca también el caso de Polonia, la República Checa y Eslovenia, que pasaron de tasas de crecimiento moderadas a tasas superiores al $5 \%$ anual.

De manera general, puede observarse que los PECOS registraron un crecimiento económico muy importante, ya que durante el periodo considerado pasaron de tener una tasa promedio de crecimiento anual negativa del $-1.9 \%$, a una tasa positiva del $6.2 \%$. Por lo anterior, parece existir evidencia de que el funcionamiento de los acuerdos de asociación no sólo sincronizaron sus economías con la UE-15, sino que las impulsaron hacia niveles de crecimiento significativos. 


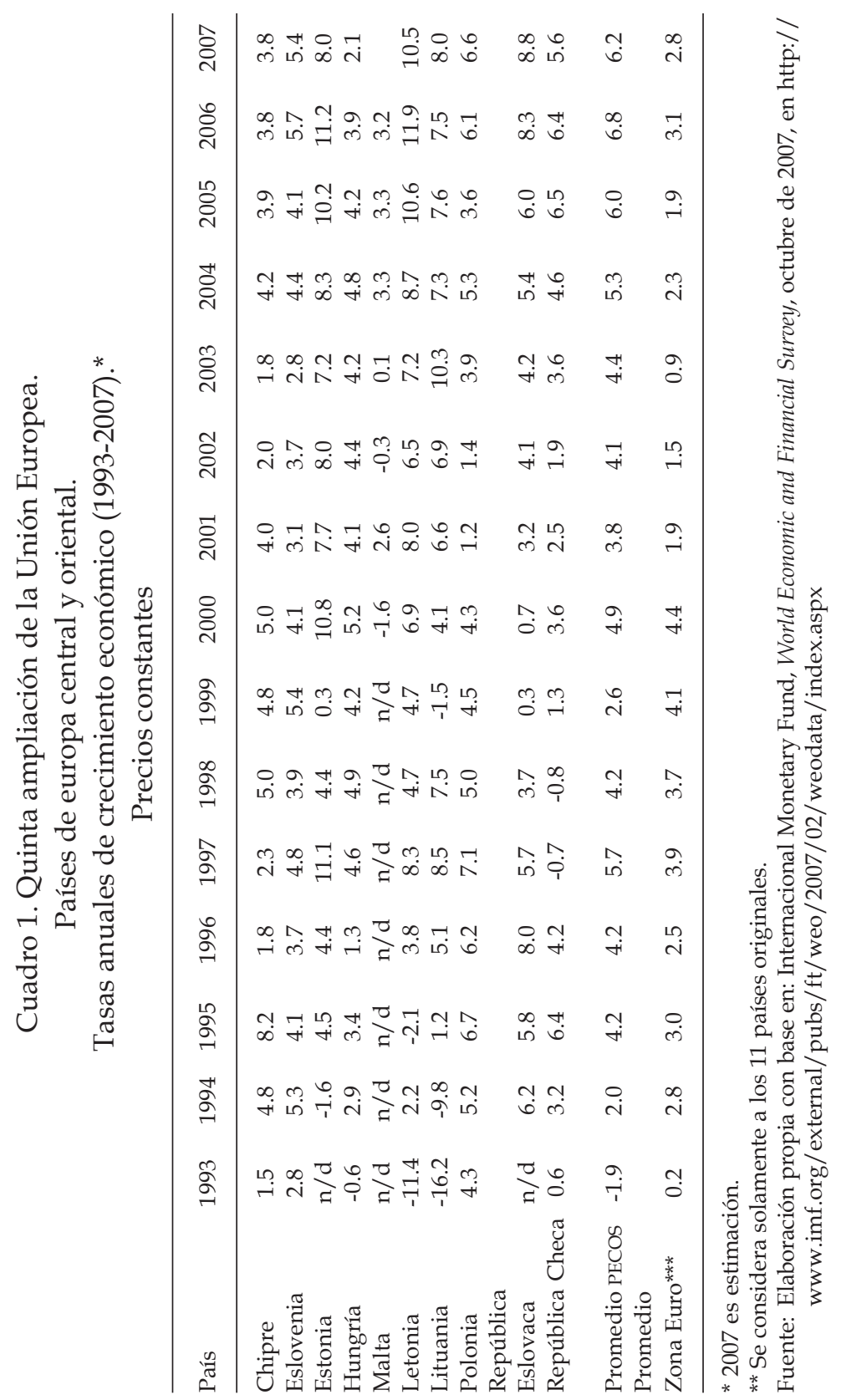


Esta apreciación concuerda con la de varios estudios que se han realizado por parte de académicos, funcionarios e instituciones internacionales para el análisis del comportamiento económico de los PECOS durante el periodo de transición hacia la economía de mercado, en el contexto de la adhesión formal a la UE-15 en el año 2004. Si bien es cierto que los problemas económicos de fondo aún persisten con intensidad variable en estos países, por lo que respecta a un crecimiento importante de sus economías en este periodo la evidencia parece ser irrefutable. ${ }^{6}$ Más aún, puede observarse que incluso los PECOS lograron en promedio tasas de crecimiento económico superiores a la UE-15.

En suma, destacamos que la quinta ampliación europea es un importante marco de referencia para otros procesos de integración económica regional en el mundo, especialmente para el TLCAN. En primer lugar, porque la estructura funcional de los acuerdos de asociación europeos y el TLCAN es similar en varios aspectos. Segundo, porque la evidencia parece indicar que ha existido un importante grado de sincronización de las economías de los PECOS a la UE-15, como también lo ha experimentado México con respecto a Estados Unidos y Canadá. Finalmente, porque ha existido un crecimiento significativo de las economías de los PECOS como consecuencia de la integración, aspecto que es de gran importancia para México si se piensa en una integración económica profunda posterior al TLCAN.

\section{Sincronización de los Ciclos Económicos en América del Norte}

\section{Antecedentes}

A pesar de que el TLCAN ha concluido su periodo de transición, a diferencia de los PECOS con respecto a la UE-15, los estudios sobre sincronización del ciclo económico de México con respecto a los Estados Unidos han cobrado interés sólo recientemente, y puede afirmarse sin temor a dudas que con respecto a la inclusión de Canadá en el análisis son prácticamente nulos.

${ }^{6}$ Véase por ejemplo: L. Johannes (2002), Y. Gulsum (2003), C. Rebolledo y E. Aguayo (2004), y World Bank (2005) entre otros. 
Para el caso de la sincronización económica entre México y Estados Unidos, Torres y Vela (2003) concluyen que la integración entre estos países se ha dado fundamentalmente a través del sector manufacturero, debido a lo cual el ciclo de negocios está prácticamente sincronizado. Para llegar a la conclusión anterior, los autores utilizan una serie de tiempo de 1990 a 2001 dividida en tres etapas: 1990 a 1993, 1994 a 1997 y 1998 a 2000, de donde se desprende que ha sido precisamente en la última cuando las economías se han integrado. La metodología empleada consiste en la estimación de coeficientes de correlación directos y cruzados sobre el componente cíclico de un conjunto de variables macroeconómicas con respecto al PIB.

Similarmente, Herrera (2004) analiza los PIBs de México y Estados Unidos para el periodo de 1993 a 2001. El autor concluye que ambas economías muestran una tendencia común y un ciclo común, debido mayormente al intenso intercambio comercial propiciado por el TLCAN. En este caso se emplean dos metodologías econométricas: para el análisis de largo plazo se considera la técnica de cointegración de Johansen (1991) y para el corto plazo la propuesta por Vahid y Engle (1993).

Otros estudios que analizan la sincronización del ciclo económico entre México y Estados Unidos lo hacen desde un punto de vista sectorial, y encuentran de la misma manera que después del TLCAN ésta se ha intensificado. Por ejemplo, Chiquiar y Ramos-Francia (2005) se enfocan fundamentalmente a las divisiones de la industria manufacturera y derivan, dentro de otros resultados, que los encadenamientos de producción y oferta entre ambos países también muestran un alto grado de sincronización en los últimos diez años; y que además se han construido fuertes y permanentes lazos de mercado intra e interindustriales.

Por su parte, Mejía et al. (2006), empleando un enfoque de ciclos económicos clásico aplicado a las cuatro grandes divisiones de la producción industrial de ambos países, establecen la existencia de asimetrías y solamente una modesta sincronización, además de que ésta es reciente y se limita a ciertas actividades.

Así, con base en los estudios realizados a la fecha, se puede concluir que existe un alto grado de sincronización entre las economías de México y Estados Unidos, sobre todo a partir del TLCAN. Sin em- 
bargo, para poder pensar en una evolución en América del Norte hacia un nivel de integración económica más profunda, es decir, hacia alguna modalidad de unión aduanera, es necesario contar con evidencia de que no solamente las economías de México y los Estados Unidos se han sincronizado, sino que también existe un alto grado de sincronización con Canadá. Como es ampliamente conocido, las economías de Canadá y Estados Unidos presentan dinámicas similares, y como se acaba de establecer, también se aprecia en el caso de México y Estados Unidos, pero, ¿cuál es el grado de sincronización entre las tres economías de América del Norte?

En esta dirección encontramos algunos estudios que proveen cierta evidencia. Por ejemplo, Cuevas et al. (2003) utilizan una metodología basada en diferentes muestras y niveles de agregación de variables económicas para establecer el grado de correlación que existe entre las tasas anuales de crecimiento. En primer lugar, deducen que para el caso de México y Estados Unidos ya existía una correlación amplia entre sus ciclos económicos con anterioridad al TLCAN, pero que se incrementó durante su periodo. Ahora bien, también señalan que dicha correlación no se registra de igual manera con Canadá como era de esperarse, aunque aumenta en alguna medida como consecuencia del TLCAN.

Similarmente, con base en una metodología de descomposición tendencia-ciclo, Fernandez y Kutan (2005) evalúan similitudes en el comportamiento de los ciclos económicos de los países de América del Norte. Los autores concluyen que la sincronización económica entre México, Estados Unidos y Canadá existía en cierta forma incluso antes de la firma del TLCAN. De esta manera, el TLCAN sólo vino a impulsar un proceso que ya se había venido gestando en el contexto de la globalización. Asimismo, Rosmy y Simons (2007), y Castillo y Ramírez (2008), establecen que existe un ciclo económico común entre Canadá, Estados Unidos y México. Más aún, los segundos autores muestran que aun antes de la firma del TLCAN ya existían movimientos comunes de largo y corto plazos entre las tres economías. En los siguientes párrafos replicamos los resultados de Castillo y Ramírez (2008), que muestran la existencia de un alto grado de sincronización entre las economías del TLCAN. 


\section{Ejercicio empírico}

Los datos que se emplearon corresponden a los PIBs de Canadá, Estados Unidos y México para el periodo 1980-2006. Las fuentes para cada país fueron, respectivamente, Statistics Canada, Bureau of Economic Analysis y el Instituto Nacional de Economía, Geografía e Informática (INEGI). Los datos corresponden a las series originales no-ajustadas por estacionalidad y se encuentran expresados en términos constantes. ${ }^{7}$ Iniciamos con una ilustración de las series en la gráfica 1. Las variables fueron transformadas logarítmicamente y la escala normalizada para facilitar la comparación entre sus comportamientos. Asimismo, en la gráfica 2 se muestran las tasas de crecimiento anual.

Resalta en primera instancia una marcada similitud en las tendencias de las series de 1995 a 2005. Cabe señalar, sin embargo, que la misma es aparente entre los años 1990 y 1994. Con respecto a las tasas de crecimiento, se notan varios episodios en los cuales las dinámicas de las series son similares. Podríamos mencionar los periodos 19801989 y de 1992 a la fecha; esto a pesar de que en 1995 la economía de México cayó significativamente; no obstante, las series siguen una dirección común. Así, con base en la gráfica 2, podríamos indicar que el único periodo en poco más de dos décadas en el que las tasas de crecimiento de los tres países no siguen una dinámica similar es de 1989 a 1992. Es claro, sin embargo, que para establecer formalmente el grado de sincronización que existe en las economías no basta con el análisis visual de las series. De tal forma que en el siguiente apartado mostramos algunos resultados econométricos que prueban que las economías de América del Norte se encuentran sincronizadas.

Para realizar la evaluación formal del grado de similitud que existe en el comportamiento de los PIBs de Canadá, Estados Unidos y México, encontramos una amplia gama de posibilidades técnicas. Podríamos, por ejemplo, emplear la metodología de Torres y Vela (2003), que se fundamenta en la estimación de correlaciones y comparación

\footnotetext{
${ }^{7}$ Notamos que el uso de series desestacionalizadas induce sesgo en las estimaciones de relaciones de cointegración y ciclo común. Ver Hecq (1998) para una discusión técnica o Herrera (2004).
} 


\section{Gráfica 1.}

Series en niveles

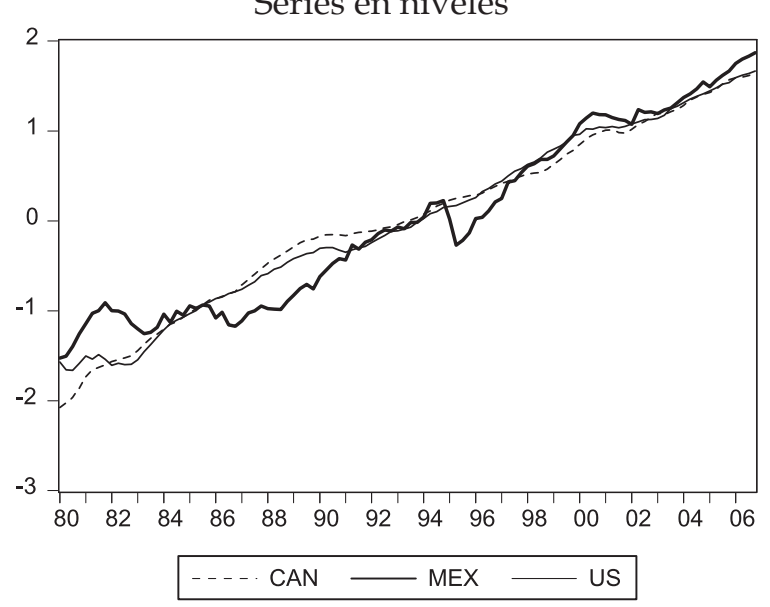

Fuente: Statistics Canada, Bureau of Economic Analysis e Instituto Nacional de Economía, Geografía e Informática (INEGI).

\section{Gráfica 2.}

Tasas de crecimiento anual

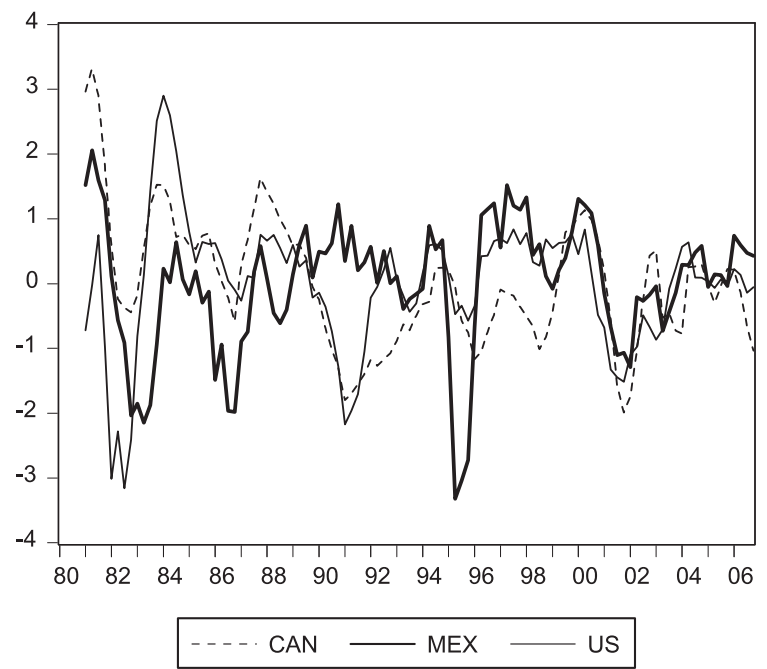

Fuente: Statistics Canada, Bureau of Economic Analysis e Instituto Nacional de Economía, Geografía e Informática (INEGI). 
de las mismas entre las series de tiempo. Sin embargo, como se indica en Chiquiar y Ramos-Francia (2005), dicha estrategia no es del todo apropiada, debido a que no puntualiza sobre el comportamiento estocástico del sistema de variables. Alternativamente, se podría llevar a cabo una descomposición tendencia-ciclo de las series por medio de un filtro o algún método de suavizamiento. A partir de este ejercicio, se llevaría a cabo una comparación de los componentes de las series para determinar el grado de similitud entre ellas. A pesar de que este procedimiento presenta una mejora con respecto al cálculo de correlaciones, la misma no impone restricción alguna en el sistema, y por ende puede producir resultados poco eficientes. En este sentido, Issler y Vahid (2001) muestran que cuando existe cointegración entre series de tiempo, resulta importante imponer restricciones de largo plazo en la estimación de ciclos comunes; de esta manera se obtienen parámetros relativamente más eficientes. Los autores sugieren la metodología de ciclos comunes propuesta por Vahid y Engle (1993) para la estimación de corto plazo. Así, anticipando la existencia de al menos una relación de cointegración entre los productos de Canadá, Estados Unidos y México, decidimos concentrarnos en esta metodología, la cual describimos a continuación.

Brevemente, partiendo de la represtación Wold de una serie de tiempo se tiene lo siguiente:

$$
\Delta y_{t}=C(L) e_{t}=C(1) e_{t}+(1-L) C^{*}(L) e_{t}
$$

Donde $y_{t}$ es un vector estacionario $n x 1$. Integrando la expresión se obtiene:

$$
y_{t}=C(1) \sum_{i=0}^{\infty} e_{t-i}+C^{*}(L) e_{t}
$$

La primera parte de esta relación representa el componente de tendencia del sistema, y la segunda el componente cíclico estacionario. Al realizar el ejercicio de cointragración se pretende obtener $\alpha^{\prime} C(1)=0$ donde $\alpha$ representa la matriz de coeficientes de cointegración. De la misma forma, para identificar la existencia de ciclos comunes se espe- 
ra que $\tilde{\alpha}^{\prime} C^{*}(L)=0$, donde $\tilde{\alpha}$ se refiere a la matriz de coeficientes de movimientos comunes (comovimientos) de corto plazo. Para encontrar $\tilde{\alpha}$, Vahid y Engle (1993) proponen una prueba sobre las correlaciones canónicas del sistema, que fundamentalmente consiste en encontrar una combinación de las series en primeras diferencias que no tenga memoria, en otras palabras, que elimine la autocorrelación entre las series. Esto se implementa realizando una prueba sobre las correlaciones canónicas al cuadrado donde la hipótesis nula es que son igual a cero. Ésta es en suma la prueba que a continuación llevamos a cabo. ${ }^{8}$

Comenzamos el ejercicio con una estimación del grado de integración de las series. Los resultados de la prueba de Kwiatkowski-PhillipsSchmidt-Shin (KPSS) se presentan en el cuadro 2. Los resultados indican que las series se encuentran integradas de orden 1 , lo cual es consistente con estudios anteriores. ${ }^{9}$

Una vez determinada la naturaleza estocástica de las series, procedemos a realizar el ejercicio de cointegración. El vector autorregresivo que consideramos incluye a los PIBs de Estados Unidos, Canadá y México en ese orden. El número de rezagos que se emplea fue determinado con criterios estándar de exclusión. Los resultados de la prueba de Johansen se presentan en el cuadro 3. De acuerdo a los estadísticos de Traza y de Valor Característico, se encuentra que existe una relación de cointegración entre las series. El vector normalizado también se muestra en el cuadro 3. Notamos que para el periodo que va de 1980 a 2006 un cambio de un punto porcentual en el PIB de Canadá se asocia con un cambio de $0.55 \%$ en el PIB de Estados Unidos; la relación con respecto al PIB de México es de $0.13 \%$. Estas asociaciones son consistentes con la teoría, que sugiere que países de similar nivel de desarrollo deben de estar relativamente más integrados que países que difieren en su estándar de nivel de vida, como es el caso de Estados Unidos y México. Más allá de la interpretación econométrica de estos resultados, es claro que conllevan a ciertas implicaciones que vale la

\footnotetext{
${ }^{8}$ Para una descripción puntual de la metodología se recomienda referirse al artículo original o a las discusiones en Herrera (2004) o Castillo y Ramírez (2008).

${ }^{9}$ Para el caso de Canadá y Estados Unidos la evidencia sobre la naturaleza estocástica del PIB es abundante. Con respecto a México ver por ejemplo Castillo y Díaz-Bautista (2003).
} 
ANA ELIZABETH JARDÓN HERNÁNDEZ/VIVIENDA PARA LOS POBRES

Cuadro 2. Pruebas de raíz unitaria

\begin{tabular}{lccc}
\hline $\begin{array}{l}\text { Series } \\
\text { PIB }\end{array}$ & Nivel & $\begin{array}{c}\text { Primera } \\
\text { diferencia }\end{array}$ & $\begin{array}{c}\text { Valor crítico } \\
5 \%\end{array}$ \\
\hline Canadá & 0.182 & 0.142 & 0.146 \\
Estados Unidos & 0.390 & 0.058 & 0.146 \\
México & 0.180 & 0.037 & 0.146 \\
\hline
\end{tabular}

Fuente: Elaboración propia.

Cuadro 3. Resultados de la prueba de cointegración

\begin{tabular}{lcccc}
\hline $\begin{array}{l}\text { Hipótesis } \\
\text { sobre } r\end{array}$ & $\begin{array}{c}\text { Estadístico } \\
\text { de traza }\end{array}$ & $\begin{array}{c}\text { Valor crítico } \\
5 \%\end{array}$ & $\begin{array}{c}\text { Máximo valor } \\
\text { característico }\end{array}$ & $\begin{array}{c}\text { Valor crítico } \\
5 \%\end{array}$ \\
\hline$r=0$ & 37.62 & 29.8 & 26.59 & 21.13 \\
$r \leq 1$ & 11.03 & 15.49 & 10.07 & 14.26 \\
$r \leq 2$ & 0.96 & 3.84 & 0.96 & 3.84 \\
& \multicolumn{4}{c}{ Vector normalizado de cointegración $(1,-0.55,-0.13)$} \\
\hline
\end{tabular}

Fuente: Elaboración propia.

pena notar. Por ejemplo, destacamos que a pesar de que en ciertos episodios las economías han mostrado comportamientos marcadamente distintos, como en 1994, en el largo plazo las tres han seguido una tendencia común. Asimismo, a pesar de que sin duda la firma del TLCAN contribuyó significativamente a promover el intercambio comercial en América del Norte, es evidente que las economías de la región seguían una dinámica similar aun antes de 1994, ya que la tendencia que comparten se inicia al menos a partir de 1980.

Condicionada a la existencia de cointegración que acabamos de determinar, ahora procedemos a instrumentar la prueba de ciclos comunes de acuerdo a Vahid y Engle (1993). Los resultados se presentan en el cuadro $4 .^{10}$

\footnotetext{
${ }^{10}$ Agradecemos al profesor Joao Issler habernos proporcionado el código de GAUSS para llevar a cabo esta prueba.
} 
ESTUDIOS FRONTERIZOS, VOL. 10, NÚM. 19, ENERO-JUNIO 2009

Cuadro 4. Prueba de ciclo común

\begin{tabular}{lcccc}
\hline $\begin{array}{l}\text { Hipótesis } \\
\text { sobre } s\end{array}$ & $\begin{array}{c}\text { Correlaciones } \\
\text { cuadradas }\end{array}$ & $\begin{array}{c}\text { Estadística } \\
\text { de cofactor }\end{array}$ & $\begin{array}{c}\text { Grados de } \\
\text { libertad }\end{array}$ & Valor $p$ \\
\hline$s>0$ & 0.023 & 2.22 & 2 & 0.33 \\
$s>1$ & 0.062 & 11.98 & 6 & 0.06 \\
$s>2$ & 0.394 & 59.54 & 12 & 0.00 \\
& & & \\
& Matriz de coeficientes de comovimiento & \\
& & & & \\
& 16.38 & 14.55 & 4.09 & \\
& -6.89 & -3.10 & 10.92 & \\
& 3.58 & -6.14 & & \\
\end{tabular}

Fuente: Elaboración propia.

De manera interesante, los resultados indican la existencia de dos ciclos comunes. La matriz de coeficientes de comovimiento también se muestra en el cuadro 4. A partir de ésta es posible determinar él o los vectores de ciclo común en el sistema. En primera instancia, y debido a que existen dos vectores, se podría normalizar con respecto al PIB de Estados Unidos y en seguida con respecto al PIB de Canadá; en ambas instancias en relación al PIB de México. En el primer caso, dividiríamos -6.14 entre 14.55 y obtendríamos un coeficiente de -0.42 . En el segundo caso, se puede considerar la división de 3.58 entre -6.89 con un resultado de -0.52. Esto implicaría que un cambio instantáneo de un punto porcentual en el producto de México, se asocia con un cambio de $0.42 \%$ en Estados Unidos y de 0.52 en Canadá. Esto es, cambios en la economía mexicana se acompañan con cambios de menor magnitud en las economías de los otros dos miembros del TLCAN; lo cual es razonable encontrar. A partir de estos coeficientes, sin embargo, podría parecer sorprendente que la magnitud del coeficiente normalizado con respecto a Canadá sea mayor al normalizado con respecto a Estados Unidos; después de todo se presume que nuestra economía se encuentra más integrada con la de Estados Unidos que con la de Canadá. En este sentido, cabe mencionar que estas magnitudes se refieren a cambios instantáneos y transitorios, no a relaciones estables de largo plazo. 
De tal forma que una manera alternativa de interpretarlos, y que pudiera ser consistente con la creencia común del grado de integración de las economías, es que los PIBs de México y Canadá son más volátiles que el de Estados Unidos. Nótese que en el caso de México el movimiento transitorio es de un punto porcentual y de 0.52 y 0.42 en Canadá y Estados Unidos respectivamente. De cualquier manera, para ser más claros en la exposición, podríamos normalizar con respecto a la variable de México, seguida de las variables de Estados Unidos y Canadá. En este caso, es posible obtener un vector como sigue: (1, -2.37, -1.92). Así, queda claro que la economía mexicana presenta una relación mayor con la economía de Estados Unidos que con la de Canadá. ${ }^{11}$

Quisiéramos también destacar que más allá de la interpretación econométrica de los resultados, lo verdaderamente interesante de este ejercicio descansa en haber identificado que las economías de América del Norte se encuentran sincronizadas, y lo han estados al menos desde 1980; es decir, no sólo comparten una tendencia común, sino también un ciclo común. Una vez más, notamos entonces que la firma del TLCAN sólo aceleró un proceso que ya se encontraba en gestación y que eventualmente de cualquier forma se iba a concretar. Mirando hacia el futuro, podemos entonces vaticinar que si las economías ya se comportaban de manera similar aun antes de 1994, ahora que ha concluido el tratado, con más razón esperaríamos que lo sigan haciendo en el futuro, de ahí la importancia de considerar hacia dónde se dirigirá la integración de las economías de América del Norte.

\section{Reflexiones finales}

\section{Lección de la experiencia de la quinta ampliación europea para el TLCAN}

En este documento se ha realizado una evaluación de la sincronización económica del periodo de pre-adhesión de los PECOS a la UE-15 y una

\footnotetext{
${ }^{11}$ La respuesta instantánea de México con respecto a Estados Unidos, 2.37, pudiera parecer elevada, sin embargo, es de hecho menor a las que se encuentran en la literatura. Por ejemplo, Herrera (2004) obtiene un coeficiente de 3.78.
} 
estimación de la misma para los países miembros del TLCAN, como casos particulares del contexto mundial de los procesos de integración económica regional. El aspecto relevante ha sido el análisis del grado de similitud que han alcanzado las economías de los países miembros del TLCAN, y se ha considerado que la sincronización económica es una premisa importante para evaluar la posibilidad de avanzar hacia una fase más profunda de integración económica regional en América del Norte, tal como ocurrió en Europa.

La principal aportación que la UE pudiera proporcionar a otros procesos de integración económica regional, sobre todo para el TLCAN, es el hecho de que el funcionamiento de los acuerdos de libre comercio propicia o impulsa de manera eficaz la sincronización de los ciclos económicos, lo cual induce a pensar que en América del Norte las condiciones económicas están ya maduras para pensar en la implementación de una etapa de intercambio comercial más avanzada del proceso de integración económica regional.

Una segunda aportación es que el impulso liberalizador del comercio propició también el crecimiento económico (incluso mayor que la UE-15), y que éste debe continuar hacia niveles de integración económica profunda. La formación de una unión aduanera es, en este contexto, el factor fundamental en la construcción del proceso. Si bien es cierto que no se considera que el proceso de integración económica regional europea sea directamente exportable, sí constituye una lección importante para América del Norte. Sin embargo, es conveniente considerar que a diferencia del TLCAN, en Europa existió desde un principio la voluntad política expresa de la integración.

La experiencia de la UE demuestra que para que se dé el funcionamiento correcto de un proceso de integración económica regional entre varios países, en términos de beneficio para sus habitantes, la clave está representada por la capacidad de avanzar más allá de la sincronización económica, hacia la construcción de instituciones dinámicas supranacionales que surgen de la voluntad política de la integración. Sin embargo, a saber, en la actualidad no existe todavía un mayor compromiso explícito en este sentido por parte de los líderes gobernantes del TLCAN. 
Lineas futuras de investigación

A pesar de los obstáculos que el TLCAN ha tenido, lo cierto es que en el año 2008 se cerró el capítulo más importante de la era moderna en las relaciones económicas de América del Norte para México. Tres son las únicas posibilidades en el futuro del TLCAN: en primer lugar, y la más improbable, es que se dé marcha atrás a lo acordado dentro de un marco referencial caracterizado por una profunda ola de renegociaciones. En segundo lugar, pudiera ser que se decidiera no hacer modificaciones significativas en materia de integración económica regional por parte de los tres países. En tercer lugar, y la más probable, es que se implemente una nueva era en la integración económica regional de América del Norte, en el contexto de una transformación hacia la unión aduanera o hacia alguna forma de ésta, como ya se mencionó.

Si se sostiene que la tercera vía de las enunciadas es la que tiene las mayores posibilidades, ya que así lo demuestra la experiencia histórica no sólo del modelo europeo sino también de otras regiones del mundo, surge por lo tanto una agenda de temas pendientes que será necesario realizar y que se considera recomendable de abordar.

En primer lugar, la línea más importante de investigación para el futuro de la integración económica regional en América del Norte tiene que ver con el análisis beneficio-costo del TLCAN, es decir, cuantificar su efecto neto. Esto deberá ser así debido a que es imprescindible identificar puntualmente a los perdedores y ganadores del proceso durante el ajuste comercial, con el objeto de canalizar los recursos asistenciales convenientes.

En este contexto, será muy importante analizar la forma como se está llevando a cabo la eliminación de las reglas de origen en América del Norte, para ciertos productos a los que ya se les ha aplicado el arancel cero. Todo parece indicar que es el preludio de una posible política de alineación arancelaria. ${ }^{12}$ También es conveniente extender la cobertura

\footnotetext{
12 Por ejemplo, el 1 de enero de 2003 entraron en vigor nuevas disposiciones con respecto a las reglas de origen para algunos productos electrónicos, al eliminarse el grado de integración nacional en los tres países. De igual forma, el 1 de enero de 2005 la disposición se extendió a varios productos del sector de alimentos y bienes industriales. En el año 2008 el Senado de la República modificó el anexo 401 del
} 
de la investigación hacia terceros países fuera del TLCAN, con el propósito de contar con un panorama completo sobre los efectos de la creación o desviación de comercio que es provocada por este tratado.

A partir de estas apreciaciones es indudable que será necesario generar estudios de beneficio-costo para México sobre la implementación de una unión aduanera formal en América del Norte. Al tratarse de una forma más avanzada de intercambio comercial de acuerdo con la teoría de la integración económica, la existencia de choques asimétricos pudiera representar costos o beneficios mucho más importantes que los generados por una zona de libre comercio.

Además, al ser una de las opciones interesantes para México en materia de integración económica profunda, el estudio de la trayectoria de crecimiento económico de los PECOS en la actualidad debe de realizarse de manera exhaustiva, y explicarse el porqué han crecido más que la UE-15. Al mismo tiempo, la generación y funcionamiento de sus instituciones supranacionales políticas, sociales y jurídicas merecen un análisis serio en el marco de referencia de la transferencia de soberanía.

Sin embargo, la formación de una unión aduanera en toda la extensión de la palabra no es ni ha sido sencilla de acuerdo con la experiencia histórica, incluso la europea, por lo que los intentos en América Latina del Mercosur, la Comunidad Andina y Centroamérica, merecen ser analizados con detenimiento. Por lo anterior, el estudio del proceso de formación de una unión aduanera para América del Norte en tres etapas, pasando de la forma más sencilla a la más compleja en función del establecimiento del arancel externo común, resulta muy interesante. En primer lugar, se tendría la alineación arancelaria por bienes; en segundo, la alineación arancelaria por sectores (unión aduanera sectorial), y en tercer lugar, la alineación arancelaria total.

Por otra parte, los resultados econométricos que se presentan sugieren también realizar estudios más profundos de la relación económica que exhiben Canadá, Estados Unidos y México. En particular,

TLCAN en lo que respecta a las reglas de origen para 23 productos. Lo anterior implica en principio el establecimiento de "una unión aduanera virtual", al aplicar a estos productos un arancel externo cero uniforme. 
sería recomendable determinar los mecanismos por medio de los cuales se gestó la sincronización de los ciclos económicos. En este sentido, podríamos proponer la evaluación del sector de la manufactura, del sector servicios y del consumo agregado como posibles factores de integración. Si bien el caso de las manufacturas ya ha sido estudiado, pensamos que un ejercicio estadístico que vaya más allá de estimar correlaciones pudiera ser de interés para la literatura. Así, queda sin duda un amplio camino por recorrer en el entendimiento del comportamiento económico de los países miembros del TLCAN. Esperamos en el futuro poder contribuir en esa dirección.

\section{Conclusión}

De manera particular, para el caso de la quinta ampliación europea, se desarrolló un amplio marco de sincronización económica derivado de la liberación de su comercio interregional en la década de los noventa, entre la UE-15 y los países adherentes. La evidencia empírica encontrada parece avalar que también ha existido una profundización en el proceso de sincronización económica en el TLCAN, ya que los resultados del análisis econométrico han identificado una relación positiva y significativa tanto a corto como a largo plazos del PIB de los tres países miembros.

Tal como ocurrió en Europa, al existir evidencia de un grado de sincronización económica avanzado, se demuestra y considera que existen las condiciones necesarias para pensar en la construcción de una etapa más profunda de intercambio comercial en América del Norte, a partir de la conclusión del periodo de transición del TLCAN. En Europa el resultado ha sido satisfactorio para los países adherentes en términos del crecimiento económico, lo cual fue posible gracias a la continuidad del proceso de integración regional hacia formas más avanzadas como la unión aduanera.

El futuro de la integración económica regional del TLCAN dependerá de condiciones macroeconómicas nacionales estables y de la voluntad política de las partes. Para profundizar el proceso, la única alternativa de acuerdo con la experiencia europea es la construcción de la unión aduanera de América del Norte. Sin embargo, esto no será 
suficiente ya que se requerirán marcos institucionales supranacionales más fuertes, como los desarrollados en Europa.

En este contexto, la tendencia debe ser generar, paulatinamente, más y mejores análisis científicos que garanticen las condiciones para una integración económica regional más profunda, que debe ir más allá del acuerdo comercial hacia una etapa de alineación arancelaria como preludio para la formación de una unión aduanera en América del Norte. Bajo esta perspectiva, la integración económica regional en América de Norte deberá ser lenta pero inexorable e irreversible.

\section{Bibliografía}

Castillo, R. y A. Díaz-Bautista (2003), “Testing for Unit Roots: Mexico's GDP", Momento Económico, 124, pp. 2-10.

y R. Ramírez (2008), "Economic Integration in North America", Applied Econometrics and International Development, núm. 8, pp. 111-122.

Chiquiar, D. y M. Ramos-Francia (2005), "Trade and Business-Cycle Synchronization: Evidence from Mexican and U.S. Manufacturing Industries", North American Journal of Economics and Finance, núm 16, pp. 187-216.

Cuevas, A., M. Messmacher y A. Werner (2003), “Sincronización macroeconómica entre México y sus socios comerciales del TLCAN", Documento de Investigación 2003-1, Banco de México.

Darvas, Z. y G. Szapáry (2004), Business Cycle Synchonization in the Enlarged EU, Magyar Nemzrti Bank, Budapest, Hungría.

Eickmeier, S. y J. Breitung (2005), How Synchronized are Central and East European Economies with the Euro Area? Evidence from Structural Factor Model, Deutsche Bundesbank, Frankfurt/M., Germany.

Fernandez, V. y A. Kutan (2005), “Do Regional Integration Agreements Increase Business Cycle Convergence? Evidence from APEC and NAFTA", Working Paper 765, William Davidson Institute.

Firdmuc, J. e I. Korhonen (2004), "A Meta Analysis of Business Cycle Correlations Between the Euro area, CEEC's and SEEC's: What do we Know?", BOFIT Discussion Papers, Bank of Finland, Helsinki. 
Gulsum, Y. (2003), “The Economic Impact of the Fifth Enlargement on the CEECs and Turkey", Treasury Journal, Special Issue, vol. 16, diciembre de 2003, Turkey, Undersecretary of the Treasury.

Guisan M., E. Aguayo y A. Carballas (2004), “Economic Growth and Cycles in Poland, Hungary, Czech Republic, Slovakia and Slovenia: A Comparison with Spain, Austria and other EU Countries, 19502002", Working Paper Series, Economic Development, núm. 79, EuroAmerican Association of Economic Development Studies, España.

Hecq, A. (1998), “Does Seasonal Adjustment Induce Common Cycles?”, Economic Letters, núm 59, pp. 289-297.

Herrera, J. (2004), "Business Cycles in Mexico and the United States: Do They Share Common Movements?", Journal of Applied Economics, núm 7, pp. 303-323.

Issler, J. y F. Vahid. (2001), "Common Cycles and the Importance of Transitory Shocks to Macroeconomic Aggregates", Journal of Monetary Economics, núm 47, pp. 449-475.

Johannes, L. (2002), "Progress in Transition: Central and Eastern Europe after a Decade of Reform", Center for Research on Economic Development \& Policy Reform Stanford University, mayo de 2002.

Johansen, S. (1991), "Estimation and Hypothesis Testing of Cointegration Vectors in Gaussian Vector Autoregressive Models", Econometrica, núm. 59, pp. 1551-1580.

Mejía-Reyes P., E. Gutiérrez y C. Farías (2006), “La sincronización de los ciclos económicos de México y Estados Unidos", Investigación Económica, núm 65, pp. 15-46.

Rebolledo, C. y E. Aguayo (2004), “Crecimiento económico en los PECOS durante la etapa de la transición", Universidad Santiago de Compostela, Ponencia presentada en la XVIII Reunión Anual. Anales de Economía Aplicada, León, España.

Rosmy, L. y D. Simons. (2007), "Is there a North American Business Cycle? An Analysis of the Period 1963-2002", Applied Econometrics and International Development, núm. 7, pp. 109-120.

Torres, A. y O. Vela (2003), "Trade Integration and Synchronization between Business Cycles of Mexico and the United States", North American Journal of Economics and Finance, núm. 14, pp. 319-342. 
Vahid, F. y R. Engle. (1993), “Common Trends and Common Cycles”, Journal of Applied Econometrics, núm. 8, pp. 341-360.

WoŸniak, P. y W. Paczyñski (2007), "Business Cycle Coherence between the Euro Area and the EU New Member States: a TimeFrequency Analysis", Center for Social and Economic Research, Polonia, julio de 2007.

World Bank (2005), Eastern Europe's Transition: Building Institutions, Presented in Economic Growth in the 1990s: Learning from a Decade of Reform, abril de 2005.

Recibido en junio de 2008 Segunda versión recibida en enero de 2009

Aprobado en febrero de 2009 